\title{
Convolution Based Compact Thermal Model Application to the Evaluation of the Thermal Impact of Die to Die Interface Including Interconnections
}

\author{
Federica Maggioni ${ }^{1,2}$, Herman Oprins ${ }^{1}$, Eric Beyne ${ }^{1}$, Ingrid De Wolf ${ }^{1,3}$, Martine Baelmans ${ }^{2}$ \\ ${ }^{1}$ IMEC, Kapeldreef 75, 3001 Leuven, Belgium \\ ${ }^{2}$ KULeuven, Department of Mechanical Engineering, Celestijnenlaan 300, 3001 Leuven, Belgium \\ ${ }^{3}$ KULeuven, Department of Metallurgy and Materials Engineering, Kasteelpark Arenberg 44, 3001 Leuven, Belgium \\ Email: maggioni@imec.be
}

\begin{abstract}
Thermal aware design of integrated circuits is essential to avoid reliability issues and failures especially in 3D-technology where active dies are placed on top of each other and more heat is dissipated on the same area available for cooling compared to conventional 2D-packages. A certain number of parameters can be selected to reduce temperature and temperature gradient. This paper is mainly focusing on the thermal impact of the dieto-die interface layer. This consists of adhesive material and arrays of interconnection structures. Besides being used for electrical die-die connections, they can be added to locally improve the low thermal conductivity of the adhesive.

Short computational time is preferred for the thermal analysis of different designs: this is why various compact thermal models (CTMs) strategies have been developed. The one presented here, for the steady state temperature evaluation of two dies stacks, is based on convolution and fast Fourier transform. A novel correction methodology is introduced to deal with the material non-homogeneity in the interface layer. Case analyses, as the interface material thermal impact or the maximum achieved temperature as a function of the amount of interconnections, are presented in the paper and can be quickly and easily performed with this CTM.
\end{abstract}

KEY WORDS: steady state, convolution, $\mu$ bump layout, thermal aware design, 3D stacks.

\section{NOMENCLATURE}

Eff $_{1} \quad$ Temperature reduction on the top die when uniform $\mu$ bump area array material properties are used instead of the underfill thermal properties $\left({ }^{\circ} \mathrm{C}\right)$.

Eff $_{2} \quad$ Temperature reduction on the bottom die when uniform underfill material properties are used instead of the $\mu$ bump array ones $\left({ }^{\circ} \mathrm{C}\right)$.

err Relative error, with respect to ambient temperature, between the FEM and the CTM results.

$\mathscr{F}$ Fourier transform operator.

$G_{k}\left(\because, z_{h}\right) \quad$ Thermal response on $z_{h}$ due to hot spot on $z_{k}$ (Green's function) $\left({ }^{\circ} \mathrm{Cm}^{2} / \mathrm{W}\right)$.

$\operatorname{HSR}_{k}\left(\because,, z_{h}\right)$ Hot Spot Response on level $z_{h}$ due hot spot on $z_{k}$ (matrix) $\left({ }^{\circ} \mathrm{Cm}^{2} / \mathrm{W}\right)$.

$\mu \mathrm{bEff}_{2} \quad$ Thermal impact of the $\mu$ bumps with respect to $T_{11, \text { und }}$ in case of two sides convection.

$P M\left(\because, z_{k}\right) \quad$ Power map on level $z_{k}\left(\mathrm{~W} / \mathrm{m}^{2}\right)$.

$T\left(\cdot,, z_{h}\right) \quad$ Temperature on level $z_{h}\left({ }^{\circ} \mathrm{C}\right)$.

$T_{k}\left(\because, z_{h}\right) \quad$ Temperature on level $z_{h}$ due to power dissipation on level $z_{k}\left({ }^{\circ} \mathrm{C}\right)$.

$T$
$\tilde{T}$

$h$

$k(\boldsymbol{x})$

$k_{\mu \text { bumps, xy }}$

$k_{\text {ubumps }, z}$

$k_{\text {underfill }} \quad$ Underfill material conductivity $\left(\mathrm{W} / \mathrm{m}^{\circ} \mathrm{C}\right)$.

$q^{\prime \prime} \quad$ Heat dissipation per unit area $\left(\mathrm{W} / \mathrm{m}^{2}\right)$.

$t \quad$ Thickness $(\mu \mathrm{m})$.

\section{Greek symbols}

$\alpha \quad$ Weights depending on the distance (-).

$\beta \quad \beta=H S R_{12} /^{(*)} H S R_{11}(-)$.

$\tilde{\gamma} \quad$ Exact weights from FEM (-).

$\gamma \quad$ Fitted weights (-).

$\rho \quad \mu$ bump array area ratio: number of $\mu$ bump cells/ total number of cells (-)

Subscripts
1
2
$\mathrm{x}_{\mathrm{i}, \mathrm{j}}$

und

$\mu \mathrm{b} \quad$ Quantity computed using the HSR with equiva-

lent $\mu$ bump array material.

Amb Ambient.

CTM Compact thermal model results.

FEM Finite elements method results.

\section{INTRODUCTION}

Integrated circuits (ICs) thermal analysis is essential to avoid reliability issues caused by high temperatures and temperature gradients [1]. The increasing demand of faster and smaller devices strengthened even more the importance of thermal models. Indeed, as miniaturization reaches its physical limits, the microelectronic industry started looking into alternative technologies such as 3D stacking where active, thinned dies are placed on top of each other. More heat is therefore dissipated over the same area available for cooling, exacerbating thermal issues.

The interface layers between two stacked dies are usually constituted by low thermal conductive, adhesive, matrix materials, referred to as underfill materials, in which interconnections, such as $\mu$ bumps [2] or $\mu$ solder balls, are integrated. These are $\mathrm{CuSn}$ or soldered structures used to allow electrical die-die connections and are usually introduced in array layouts (Fig. 1). Since the $\mu$ bumps have a much higher thermal conductivity than the surrounding material, they can be inserted merely on 


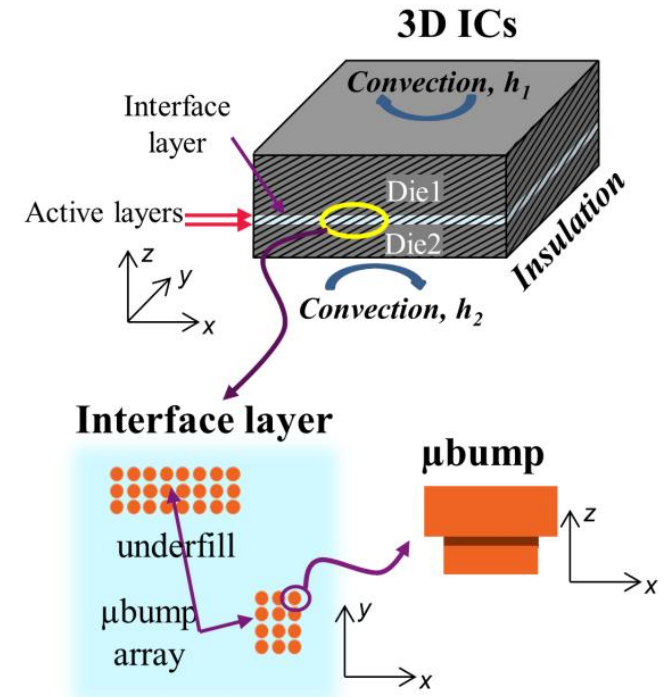

Fig. 1 Structure of the die stack, of the interface layer and of the single $\mu$ bump.

thermal basis: in this case they are referred to as dummy $\mu$ bumps or thermal $\mu$ bumps. However, due to the high additional cost, their number and placement should be thermally optimized.

In addition to the $\mu$ bump layout, the design engineers can select the position and the intensity of the heat dissipating structures and the values of certain design parameters such as the thickness of the layers, the interface material and the convection coefficients. The thermal behaviors of different configurations have to be tested and compared, through modeling techniques, in the early design phase, already in the pathFinding phase $[3,4]$, to select the best technology and design options. Finite elements methods (FEMs) are commonly used for this aim. On the one hand they provide accurate simulations, on the other hand they require high computational time and specific modeling expertise. This is the reason why different compact thermal model (CTM) methodologies have been developed in the last decades.

These CTMs can be categorized into four different groups depending on the used strategy. The original approach is based on the electrical-thermal analogy in which the thermal system is schematized as a resistance-capacitance (RC) network [5-8]. In this framework, either low resolution temperature profiles are considered, or large finite difference problems have to be solved. Liquid cooling compact thermal modeling, which is outside the scope of this paper, has also been developed following this strategy [7]. The second method tries to find an analytical solution to the heat transfer equation. However, this is possible just for drastically simplified situations [9]. The third group of solutions relies on the mathematical theory of model order reduction in which the system of equations, obtained by FEM, is projected into lower dimensional spaces, using various projecting techniques, before being solved [10-13]. This class of methods relies on a full FEM simulation for each specific structure and design, requiring, therefore, modeling expertise by the end user and computationally expensive preprocessing operations. Just the impact of different power maps can be easily analyzed without the need of new FEM models. The last method exploits the Green's function theory and the superposition principle [14-19]. Each of these methodologies has its pros and cons but they all mainly fail to include the $\mu$ bump impact in a fast and user friendly way. One of the main difficulties to achieve this is caused by the non-local $\mu$ bumps impact leading to a thermal effect that is traceable throughout the whole stack. Moreover, even if homogenization techniques [20] can be used within each single $\mu$ bump array to retrieve equivalent material properties, they cannot be exploited globally. This is because the $\mu$ bumps are uniformly distributed within the array but not on the whole interface layer.

The model presented in this paper belongs to the last of these groups. It applies the Green's function theory through convolutions between the temperature responses to individual hot spot (HS) power dissipations and the actual dissipated power (in matrix form) [19,21]. Fast Fourier transform is employed to further speed up the computational time. This method is really powerful if the heat path is independent of the heat dissipation position but, unfortunately, this hypothesis is never fulfilled in practical applications. One reason can be identified in the finite size of the stack itself since the lateral boundaries, assumed to be insulated, modify the heat path. This issue is solved using a technique retrieved from the electrical analogy: the method of images $[14,19]$. Another reason is the material non-uniformity in the interface layer: the inclusion of particular $\mu$ bump layouts deviates the heat path towards more conductive regions. A novel correction methodology has been developed to deal with this issue in case of two equally sized, face-to-face die stacks [21]. The temperature profiles on the two active layers are computed as weighted average between the two convolution results obtained considering homogeneous interface material (underfill and full $\mu$ bump array). The weights are calculated from previously established fitting models and they take into account the selected cooling strategy, the stack dimensions, the material properties (referred to as system parameters) and the particular $\mu$ bump layout (in matrix form).

The model has been validated against FE results obtained for the same structure: a two die stack with equivalent convective boundary conditions. Good agreement (less than 2\% error) and considerable speed up (30 times faster) has been proved. Moreover, one of the main advantages of this methodology is that the thermal impact of different parameters can be easily checked by just changing entries in matrices (dissipated power and $\mu$ bumps locations) or the values of some input numbers (system parameters). Its flexibility in thermally comparing different scenarios, in terms of power dissipation and $\mu$ bumps layouts, makes the model interesting for the design phase. Differently from previously developed strategies, almost no limitations are imposed on the $\mu$ bumps layouts and the thermal effect of the in-plane inhomogeneity in the interface layer is captured both locally and globally. Moreover, this methodology can be exploited to model both low power and high power devices.

Examples of two typical applications are given in the following of this paper. One example concerns the study of the thermal impact of different interface materials, the other example deals with the relationship between the maximum temperature and the amount of included $\mu$ bumps. Since, as already stated, the amount and the placement of $\mu$ bump arrays should be optimized in terms of temperature and cost, this last application of the CTM allows a quick and easy way to evaluate if, 
Table 1. System parameters and their ranges for which the model is valid.

\begin{tabular}{l|c|c}
\hline \multicolumn{1}{c|}{ Parameter } & Minimum & Maximum \\
\hline$h_{1}\left(\mathrm{~W} / \mathrm{m}^{2} \mathrm{~K}\right)$ & 0 & 15000 \\
$h_{2}\left(\mathrm{~W} / \mathrm{m}^{2} \mathrm{~K}\right)$ & 0 & 15000 \\
$h_{1}+h_{2}\left(\mathrm{~W} / \mathrm{m}^{2} \mathrm{~K}\right)$ & 500 & 15000 \\
$\mathrm{k}_{\mu \text { bumps } \mathrm{z}(\mathrm{W} / \mathrm{mK})}$ & 2.5 & 20 \\
$\mathrm{k}_{\mu}$ bumps,xy $(\mathrm{W} / \mathrm{mK})$ & 0.3 & 13.5 \\
$\mathrm{k}_{\text {underfill }}(\mathrm{W} / \mathrm{mK})$ & 0.2 & 9 \\
Top die thickness $(\mu \mathrm{m})$ & 20 & 400 \\
Bottom die thickness $(\mu \mathrm{m})$ & 20 & 400 \\
Interface layer thickness $(\mu \mathrm{m})$ & 2 & 30 \\
$\rho$, area ratio & 0.09 & 0.5 \\
\hline
\end{tabular}

how many and where $\mu$ bumps should be preferably included. No modeling expertise is required by the end user: it is sufficient to supply the input parameters together with the power and $\mu$ bump layout information in the corresponding matrices.

The remaining of the paper is organized as follows: first the assumptions underlying the model are listed, then the basic CTM and its generalization to include the $\mu$ bumps impact are explained and finally, before conclusions, the results of the two practical applications are shown.

\section{MODEL ASSUMPTIONS}

The model has been developed to analyze the steady state for stacks of two $8 \mathrm{~mm} \times 8 \mathrm{~mm}$ dies. Since the active regions, on the top of the bottom die and on the bottom of the top die (faceto-face set-up), have a thickness in the order of nm, they are assumed to be planar (Fig. 1). The interface layer in between the two dies consists of $\mu$ bumps surrounded by underfill material. The interconnections are not treated individually but as arrays. Individual dimension and pitch are used to obtain equivalent material properties. In this model, just two material sets are allowed in the interface layer and they are referred to as underfill and $\mu$ bumps material. The location and the dimensions of the $\mu$ bump arrays, as well as the intensity and the position of the heat dissipation areas, are stored in matrices and can be freely chosen by the user.

Since the heat removal strategies are mainly on top and bottom of the stack (PCB, heat sink, heat spreader,...) and since the horizontal boundary area is much larger than the vertical one, insulation is assumed on the lateral sides of the die stack. Equivalent convective boundary conditions are imposed on the top and bottom boundaries through constant coefficients, $h_{1}$ and $h_{2}$ respectively. All the system parameters can be freely chosen by the user but, if particular $\mu$ bump layouts are assumed in the interface layers, their values should be within the ranges reported in Table 1. Also the area ratio $\rho$, that is the fraction of the interface area covered by $\mu$ bump arrays, has to be selected within a certain range.

\section{BASIC METHODOLOGY}

The governing heat transfer equation for planar heat dissipation can be written as

$$
\nabla[-k(\boldsymbol{x}) \nabla T(\boldsymbol{x})]=q^{\prime \prime}(\boldsymbol{x})
$$

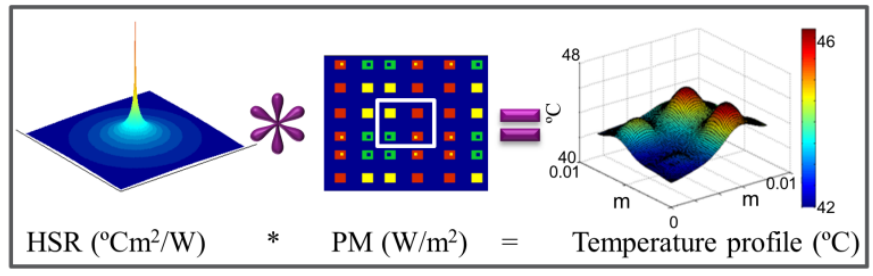

Fig. 2 CTM basic methodology: the convolution between the HSR (left) and the extended PM including images (center) gives the temperature profile (right).

where the temperature independent thermal conductivity $k(x)$, the temperature $T(\boldsymbol{x})$ and the dissipated power density $q^{\prime \prime}(\boldsymbol{x})$ depend on the position $x=(x, y, z)$. If, in a first approximation, a uniform interface material (no specific $\mu$ bump layout) is considered, i.e. $k(\boldsymbol{x})=k(z)$, then the Green's function theory applies. This means that the temperature profile on level $z=z_{h}$, due to heat dissipation per unit area $q^{\prime \prime}\left(x, y, z_{k}\right)$ on $z=z_{k}$, can be computed as the convolution between the temperature response on level $z_{h}$ due to HS power dissipation on level $z_{k}, G_{k}\left(x, y, z_{h}\right)$, and the actual dissipated power on $z_{k}$, $q^{\prime \prime}\left(x, y, z_{k}\right)$

$T_{k}\left(x, y, z_{h}\right)=\int G_{k}\left(s, t, z_{h}\right) q^{\prime \prime}\left(x-s, y-t, z_{k}\right) d s d t=G_{k} * q^{\prime \prime}$.

The validity of this equation is restricted to situations where the $G_{k}$ functions are independent of the $(x, y)$ position in which the HS is generated. The finite horizontal size of the stack causes this hypothesis to fail but, since insulation is assumed on the lateral boundaries, the method of images $[14,19]$ can be employed to allow the application of the Green's function method. More precisely, the $G_{k}$ functions are computed assuming a stack of infinite horizontal size and the $q^{\prime \prime}$ functions are enlarged including mirrored images before convolution is performed. Theoretically, the number of images needed to model insulation should be infinite but, due to the decay of $G_{k}$, a proper finite number can be assumed [19].

To numerically compute the temperature profiles, both the $G_{k}$ and the extended $q^{\prime \prime}$ functions have to be discretized over grids with the same mesh size and they will be referred to as hot spot responses (HSRs) and power maps (PMs) respectively (Fig. 2). The HSRs are computed via FEM and, in case of two dies stacks, four of them need to be calculated.

The numerical solution of equation (2) involves a double summation having, therefore, $O\left(N^{2}\right)$ complexity where $N$ is the number of elements in the extended PM. The application of the fast Fourier transform (FFT) algorithm, allowing the computation of the convolution integral as the inverse Fourier transform of the element-by-element product of the transformed matrices, significantly reduces the computational effort up to $O(N \log N)$. This procedure allows for more than 70 times reduction in computational time in comparison with FEM [19].

\section{INTERCONNECTIONS THERMAL IMPACT}

Another characteristic of the IC stack, which prevents the requirement of a position independent heat path to be fulfilled, is the inclusion of particular $\mu$ bump layouts in the interface layer since, in this way, its thermal conductivity becomes nonuniform. Moreover, since the thermal effect of the material heterogeneity is global, a local correction approach limited to the 


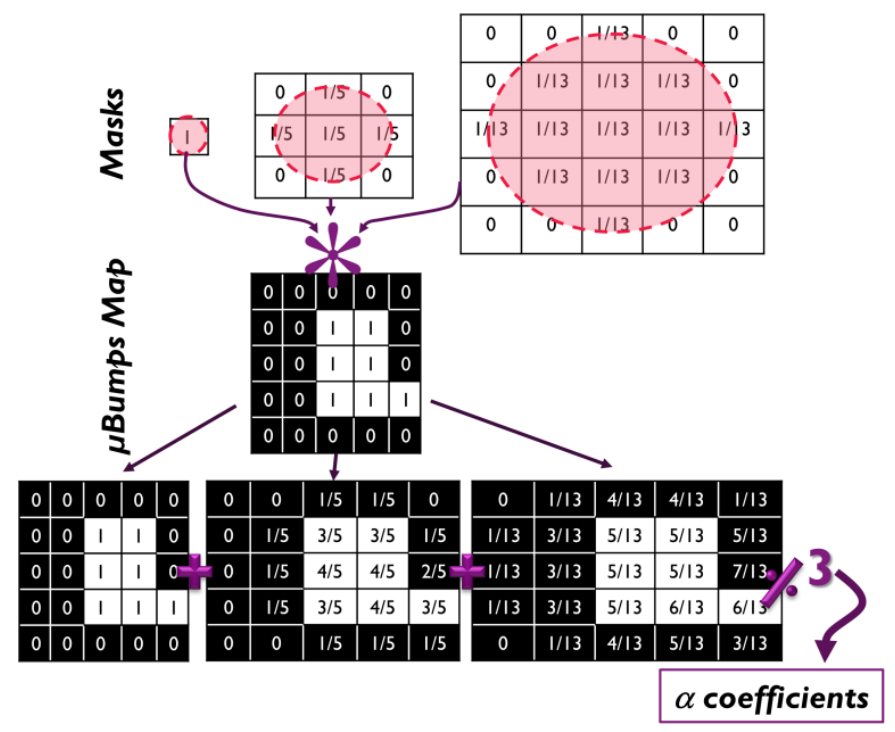

Fig. $3 \alpha$-weights computation technique.

discontinuity regions is not enough to properly describe the temperature profiles.

To ensure the HSRs to be position independent, and to exploit the power of the FFT convolution technique, they are computed considering uniform material in between the dies: for two dies stacks, eight different HSRs are, therefore, required. For each interface material (underfill or $\mu$ bumps material), indeed, the thermal response of HS powering one of the two active layers needs to be monitored for both layers. The temperature profiles obtained, by convolution, for the different interface materials are, then, combined to take into account specific $\mu$ bump layouts [21].

The correction methodology, which will be briefly described in the rest of this section, starts considering a simplified situation in which uniform power is dissipated on the top die and insulation is imposed on the top of the stack. Generalizations to more practical situations, with general heat dissipation on both dies and convective boundary conditions on top and bottom side of the stack, follow.

\section{Fitting procedure for temperature on top die}

A weighted average between the temperatures obtained using, in the convolution operations, the HSRs for underfill and $\mu$ bumps materials only, $\left(T_{11, u n d}\right.$ and $\left.T_{11, \mu b}\right)$, is initially used to estimate the temperature on the top die

$$
\tilde{T}_{11}=\alpha T_{11, \mu b}+(1-\alpha) T_{11, \text { und }} .
$$

The subscripts refer to the HS dissipation layer and the temperature computation layer respectively; 1 indicates the top die and 2 the bottom die. When, in the following of the paper, only one digit is used, it refers to the temperature computation layer and the impact of the total heat dissipation is considered.

The weights, $\alpha(x, y)$, are position dependent. They take into account both the local and the global $\mu$ bump locations. The interconnection layout is stored into a binary matrix, named $\mu$ bumps map, with the same resolution as the original PM; 1's entries represent part of $\mu$ bump arrays while 0 's indicate underfill material. A calculation methodology based on the average of numerous convolutions, between masks of increasing size but unitary sum and the $\mu$ bumps map, is used to compute the $\alpha$-weights and it is illustrated in Fig. 3 .
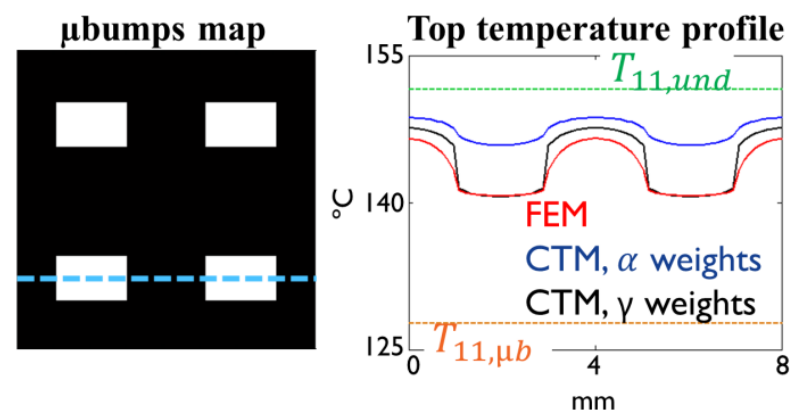

Fig. 4 Left: $\mu$ bumps map (black=underfill, white $=\mu$ bumps) and location of the cross sections shown on the right. Right: temperature profiles on the horizontal line: FEM (red), CTM with $\alpha$-weights (blue), CTM with $\gamma$-weights (black), $T_{11, u n d}$ (green) and $T_{11, \mu b}$ (orange).

However, this technique is not enough to fully explain the complex thermal effect of heterogeneous interface materials. For this reason, a fitting approach based on numerous FEM simulations is undertaken. The top temperature profile, $T_{1, F E M}$, obtained via FEM for uniform power dissipation on the top die, is recorded for various system parameters values and $\mu$ bump layouts covering the ranges in Table 1 . From these data the exact weights, $\tilde{\gamma}$, needed in the average are computed, i.e. $T_{1, F E M}=\tilde{\gamma} T_{11, \text { und }}+(1-\tilde{\gamma}) T_{11, \mu b}$, and fitted against the original $\alpha$-weights. The fittings are initially performed grouping all the temperatures data obtained for the same values of the system parameters, of the $\mu$ bump area ratio, $\rho$, and of the cell-type (underfill or $\mu$ bumps). Quadratic polynomials are used to fit the data referring to underfill cells $\left(\gamma=a \alpha^{2}+b \alpha+c\right)$ while linear ones are sufficient for $\mu$ bumps cells $(\gamma=d \alpha+e)$. Five overall quadratic fitting models of the polynomial coefficients, $a, b, c, d, e$ with respect to the system parameters and the area ratio are finally computed allowing the direct computation of the $\gamma$-weights from the input parameters values and the $\mu$ bump layout. The top temperature can, therefore, be computed as

$$
T_{11}=\gamma T_{11, \text { und }}+(1-\gamma) T_{11, \mu b} .
$$

The results are shown in Fig. 4: the left plot represents the $\mu$ bumps map (black $=$ underfill, white $=\mu$ bumps) and the indication of the location of the temperature cross-section on the right. In the other graph, the green line indicates $T_{11 \text {,und }}$ while the orange one $T_{11, \mu b}$, red curve is for the FEM result, blue for the $\alpha$-weight CTM and black for the $\gamma$-weight CTM: the improvement got throughout the fitting model is clearly visible.

\section{Temperature on the bottom die}

Since in the simplified situation with a thermally insulated top side of the stack, the heat path is from top to bottom, whatever uniform material is used in the interface layer, the temperature computed on the bottom die has the same value, i.e. $T_{12, \text { und }}=T_{12, \mu b}$. This is because, in such a situation, the heat path is vertical and only what comes after the computational level has an impact on the temperature value. A schematic illustrating this situation in terms of resistance network [5] is shown in Fig. 5. However, if a specific $\mu$ bump layout is introduced, the heat path is no longer vertical and non-uniform temperature profiles are observed on the bottom die. Nevertheless, no combination of $T_{12, \text { und }}$ and $T_{12, \mu b}$ can ever result in nonuniform profile. 


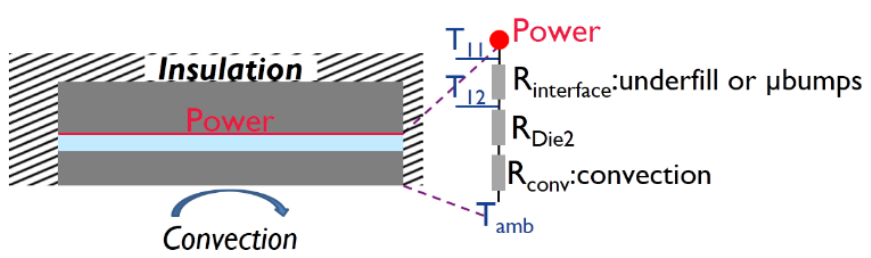

$T_{11}-T_{\text {amb }}=P\left(R_{\text {interface }}+R_{\text {Die2 }}+R_{\text {conv }}\right) ; T_{11}-T_{12}=P\left(R_{\text {interface }}\right)$ $\Rightarrow T_{12}-T_{\text {amb }}=P\left(R_{\text {Die2 }}+R_{\text {conv }}\right)$, independent of interface material

Fig. 5 Schematic of the reason why $T_{12, \text { und }}=T_{12, \mu b}$.

$T_{12}$ is, therefore, computed starting from the result obtained on the top die, $T_{11}$. Let's suppose to have uniform interface material and let's define $\beta=H S R_{12} /^{(*)} H S R_{11}$, where $/^{(*)}$ indicates the inverse convolution operator, then $T_{12}$ can be obtained as

$$
T_{12}=P M_{1} * H S R_{12}=P M_{1} * H S R_{11} * \beta=T_{11} * \beta .
$$

This formula can still be used for non-uniform interface material but, in this case, there are $H S R_{12, \text { und }}, H S R_{12, \mu b}, H S R_{11, \text { und }}$ and $H S R_{11, \mu b}$. Thus, initially, two temperature profiles $T_{12, \text { und }}$ and $T_{12, \mu b}$ are computed using, respectively, the underfill and the $\mu$ bumps HSRs in equation (5). They are, then, combined considering the underfill results in correspondence with underfill cells and the $\mu$ bumps results elsewhere. A final smoothing step is needed since the curvature of the temperature of the top die is opposite to the one of the bottom die (Fig. 6 left).

\section{Convection on both sides}

When convective boundary conditions are applied both on top and bottom of the stack and uniform power is dissipated on the top die, the thermal impact of the type of uniform material used in the interface layer is detectable both in the top and the bottom temperature profiles (Fig. 6 right). The thermal effects of using different uniform interface materials are defined as $\mathrm{Eff}_{1}=T_{11, \text { und }}-T_{11, \mu b}$ on the top die and $\mathrm{Eff}_{2}=T_{12, \mu b}$ $T_{12, \text { und }}$ on the bottom one. If a particular $\mu$ bump layout is introduced, the temperature on the top die in the $\mu$ bump location, for example, can be lower than $T_{11, \mu b}$. This means that, starting from $T_{11, \text { und }}$ as reference, the real thermal effect is higher than Eff $_{1}$. From a fitting procedure of numerous FE models in which the thermal impacts, due to square $\mu$ bump arrays of different

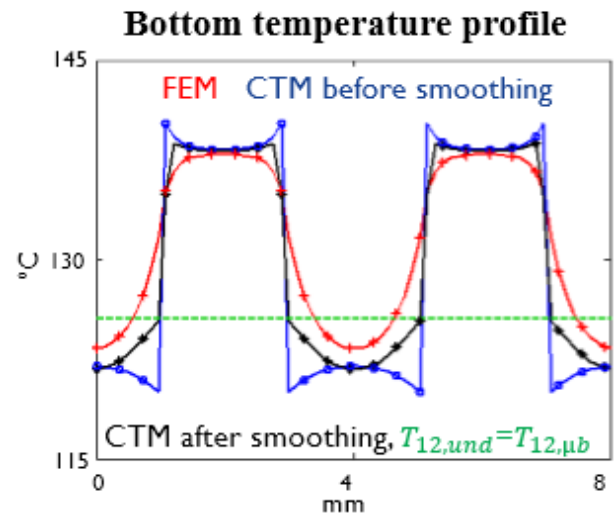

dimensions, have been recorded for various values of the convection coefficients $h_{1}$ and $h_{2}$, the following relationship has been established for the top die $\mu$ bumps effect [21]

$$
\mu \mathrm{bEff}=\mathrm{Eff}_{1}+\left(1-\rho^{1 / 4}\right) \mathrm{Eff}_{2} .
$$

From equations (4) and (6), the temperature profile on the top die can, therefore, be computed as

$$
T_{11}=T_{11 \text {,und }}-(1-\gamma) \mu \mathrm{bEff}
$$

where the $\gamma$ coefficients are the ones obtained using the fitted models for a configuration insulated on top and with a convection coefficient on bottom $h=h_{1}+h_{2}$. The temperature on the bottom die is, afterwards, computed following the same procedure previously illustrated.

\section{Power dissipation on the bottom die}

In case power is dissipated on the bottom die, exactly the same procedure illustrated here is applied just assuming the stack to be flipped upside-down. If power is dissipated both on top and bottom die, the temperature increments due to $P M_{1}$ and $P M_{2}$ are computed separately and, afterwards, exploiting superposition principle, they are summed up

$$
T_{1}=T_{11}+T_{21}, T_{2}=T_{12}+T_{22} .
$$

\section{Non uniform power dissipation}

In case of non-uniform heat dissipation, the specific PMs are substituted to the uniform ones in all the previous steps except in the $\gamma$ calculation. Anyway, since these weights are obtained considering uniform heat dissipation, artificial effects appear in particular where little or no power is produced and a boundary between underfill and $\mu$ bumps material is present. This is why, in low power locations, the $\mu$ bumps effect in eq. (7) is weighted by taking into account how much power is dissipated in each location and in its neighborhood, following a convolution procedure similar to the one illustrated in Fig. 3 for the computation of the $\alpha$-weights.

Some results for the most generic case with two side convection and non-uniform PMs are shown in Fig. 7. In the right column, the considered PMs and the $\mu$ bumps map are shown. The temperature diagonal cross sections on top and bottom die are plotted on the left column: blue indicates CTM results, red FEM, black is used for a structure with homogeneous underfill material and green for homogeneous $\mu$ bumps material. All the

\section{Material impact, convection top/bottom}

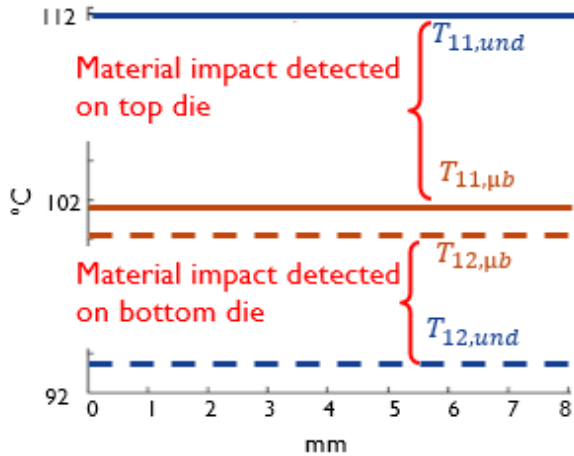

Fig. 6 Left: cross section of the bottom temperature profiles for the same situation in Fig. 4; FEM (red), CTM before smoothing (blue), CTM after smoothing (black) and $T_{12, u n d}=T_{12, \mu b}$ (green). Right: thermal impact of uniform interface material in case of convection on both sides. 


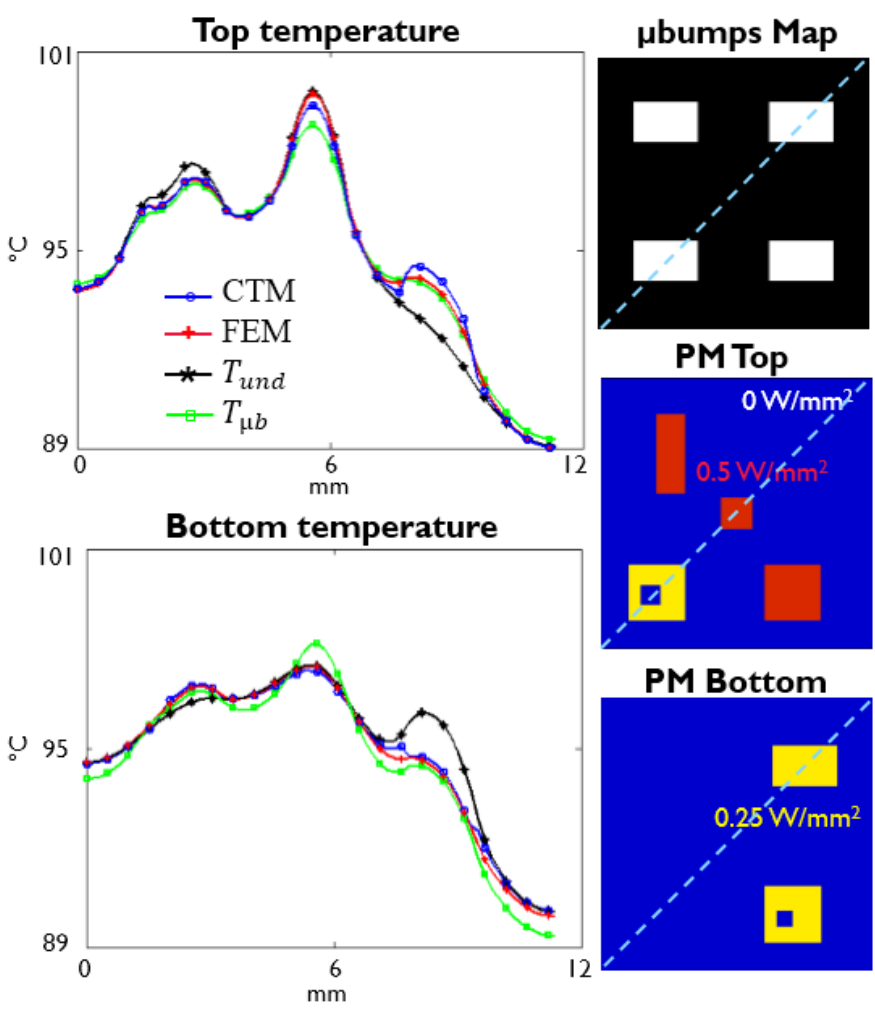

Fig. 7 Left: temperature on top and bottom die for a general case with convection on both sides and non-uniform PMs: CTM (blue), FEM (red), all underfill (black) and all $\mu$ bumps (green) solutions are shown. Right: $\mu$ bumps map, PMs and the cross section location.

results refer to models of the die-interface-die structure, as illustrated in Fig. 1; package, PCB, heat sink,... are not included in any of them but are taken into account by the equivalent convective boundary conditions. The accuracy of the CTM that considers the $\mu$ bump effect is higher than the ones achieved by the models with uniform interface material. This shows that this CTM is able to capture the global and local effects of particular $\mu$ bump layouts. The maximum error, defined as

$$
e r r=\frac{T_{F E M}-T_{C T M}}{T_{F E M}-T_{A m b}},
$$

results to be less than $1 \%$ and the computational time goes down to $10 \mathrm{sec}$ on a desktop PC, 30 times faster than FEM.

\section{RESULTS AND APPLICATIONS}

One of the main characteristics of this compact thermal model is its ease of use even without having specific modeling expertise. Once the HSRs have been computed, the impact of different materials, dimensions, cooling strategies, $\mu$ bump layouts and power distribution can be tested and studied by just modifying input numbers or entries in matrices.

\section{Material impact}

As a first illustration of the capability of the CTM, an analysis of the thermal impact of the interface materials is performed. Different underfill materials and different $\mu$ bumps equivalent properties, obtained modifying individual $\mu$ bump pitches and dimensions, named as Case1, Case 2 and Case3, are considered and listed in Table 2 together with all the other system parameters (kept constant). The $\mu$ bump layout consists in
Table 2. Parameters values used in the study of the thermal impact of the interface material.

\begin{tabular}{|c|c|c|c|}
\hline & Case1 & Case2 & Case3 \\
\hline$k_{\mu b u m p s, x y}(\mathrm{~W} / \mathrm{mK})$ & 1.3 & 1.6 & 2 \\
\hline$k_{\mu b u m p s, z}(\mathrm{~W} / \mathrm{mK})$ & 5 & 5 & 7 \\
\hline$k_{\text {underfill }}(\mathrm{W} / \mathrm{mK})$ & 0.7 & 1 & 1.5 \\
\hline$h_{1}$ & & $3000 \mathrm{~W} / \mathrm{m}^{2} \mathrm{~K}$ & \\
\hline$h_{2}$ & & Insulation & \\
\hline$t_{1}$ & & $200 \mu \mathrm{m}$ & \\
\hline$t_{2}$ & & $50 \mu \mathrm{m}$ & \\
\hline$t_{\text {interface }}$ & & $13 \mu \mathrm{m}$ & \\
\hline$T_{A m b}$ & & $25^{\circ} \mathrm{C}$ & \\
\hline Mesh size & & $120 \mu \mathrm{m}$ & \\
\hline Chip size & & $8.16 \times 8.16 \mathrm{~mm}^{2}$ & \\
\hline
\end{tabular}

a configuration based on the commercial Wide-IO set-up according to the JEDEC standard [22]. This standard defines the features and layout of the interconnections between logic and memory chips. Additional thermal $\mu$ bumps are added in correspondence with the heat dissipation position but they cover a slightly smaller area than the high power one. The $\mu$ bumps map and the PMs are kept constant (Fig. 8) and no power is dissipated on the top die.

The top and bottom temperatures on the diagonal cross section for the three different cases are shown on the left of Fig. 9; Case 1 is represented by blue, Case 2 by red and Case 3 by green curves. The graphs clearly show the impact of the interface material on the temperature values. Since the power is dissipated on the bottom die and the heat is convectively removed from the top, the positive effect of using better conductive material is clearly visible in the bottom temperature profile. From Case1 to Case 3 , indeed, a $3.5 \%$ reduction on the maximum temperature (relative to ambient) is experienced. The use of better materials in the interface layer has, however, an impact also on the top temperature and, due to the improved thermal conductivity from Case 1 to Case3, this results in a slight increment of the top temperature $(0.15 \%$ relative to ambient $)$.

The right column of the same graph shows the complete top and bottom temperature profiles obtained with Case 3 materials. Even if some discontinuities are visible in the boundary regions between underfill and $\mu$ bumps material, previously performed model validations with respect to FE results always showed errors, as defined in eq. (9), smaller than $2 \%$, which are highly acceptable.

Through this analysis, that can be easily performed changing some input numbers, it is possible to rapidly obtain information about the improvements in terms of temperature, and

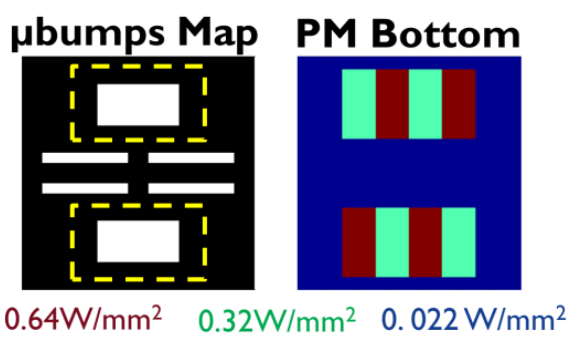

Fig. $8 \mu$ Bumps map and bottom power map used in the study of the interface material thermal impact. Yellow lines indicate the high power dissipation area. 

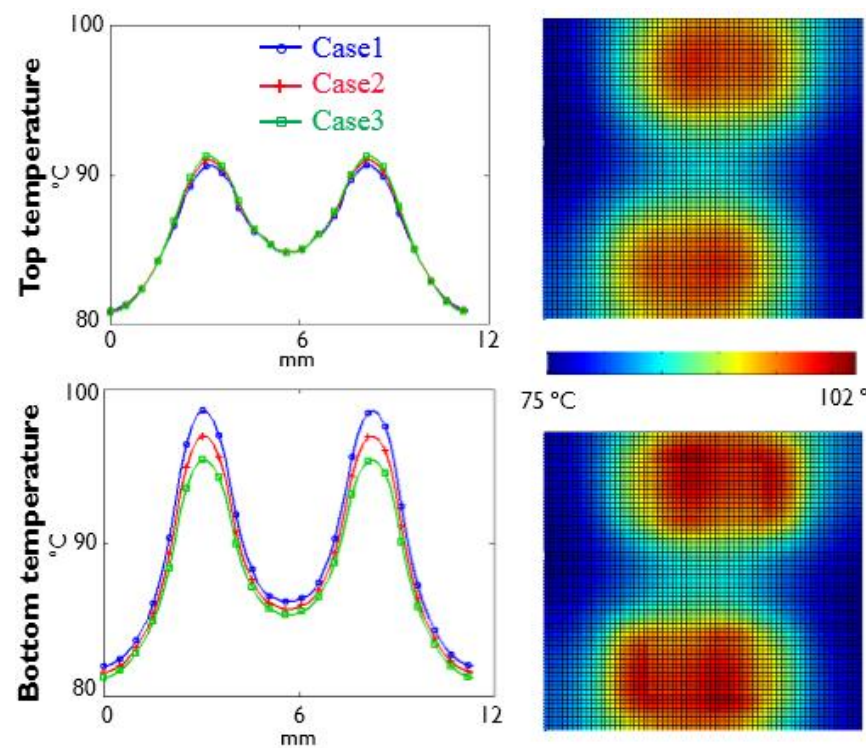

$75^{\circ} \mathrm{C}$

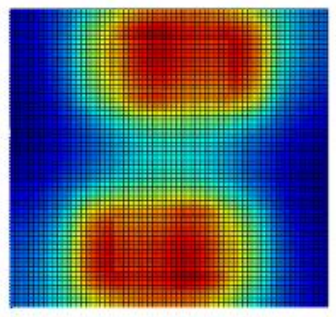

Fig. 9 Left: comparison of the temperature profiles obtained for Case1 (blue), Case 2 (red) and Case3 (green) material on the diagonal of the top and bottom die. Right: complete temperature profiles on top and bottom die for Case 3 material.

therefore, reliability, achievable using better interface materials. This study can also help to decide whether, taking also into account the higher price for better material, the employment of more conductive material is beneficial or not.

\section{$\mu$ Bump layout thermal impact}

Another important early design phase question concerns the amount and the location of the dummy $\mu$ bumps. They are included in the interface layer just to improve the thermal performances of the device and not on an electrical connection basis: for this reason they can be positioned with a higher design freedom. However, the final price of the chip increases with the amount of included $\mu$ structures and, therefore, an accurate analysis of the involved costs versus performances has to be carried out. The CTM presented in this paper is well suited for this analysis since it allows for the rapid and easy computation of the thermal impact of different $\mu$ bump layouts.

The study presented here relates the $\mu$ bump area ratio, $\rho$, to the temperature in the middle of the heat dissipation area. This is, in most cases, the location of the maximum temperature. Fig. 10 shows the considered $\mu$ bumps maps: the basic layout is constituted by the commercial Wide-IO configuration. Rectangular arrays of increasing size, centered with the high power dissipation areas, are added. The basic cases of homogeneous underfill and $\mu$ bumps materials are also considered. The last plot in the same figure shows the bottom PM; the red cross indicates the location to which the data in Fig. 11 refer. No power is dissipated on the top die while the values of the material conductivities and of the other system parameters are the one used in Case1 in the previous paragraph (Table 2).

The results of this study are presented in Fig. 11 where the bottom temperature in the defined location is shown as a function of the $\mu$ bump area ratio, $\rho$; red circles refer to CTM results while blue crosses to FEM ones. Some significant cases are highlighted. The graph shows the positive trend in temperature reduction gained by introducing extra dummy $\mu$ bumps centered

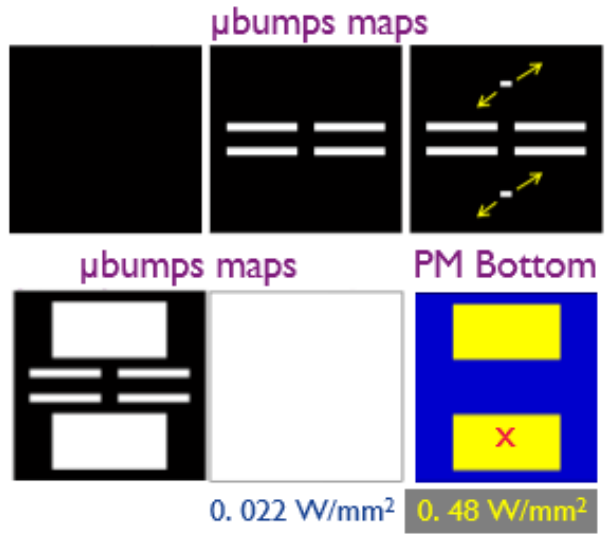

Fig. $10 \mu$ Bump layouts used in the study of the interconnections thermal impact (Fig. 11). The last plot shows the PM used on the bottom die and the red cross indicates the position to which the data in Fig. 11 refer to. No power is dissipated on top.

above the heat dissipation area. The thermal effect of the WideIO layout is minimal since its location is not aligned with the heat sources. As soon as some $\mu$ bumps are placed aligned with the hot region, the temperature starts dropping. The temperature reduction trend, however, saturates after a while: when $\rho$ reaches the value of $0.3-0.4$, which corresponds to the covering of the whole heat generating area, no extra improvement is achieved. The addition of extra dummy $\mu$ bumps to the WideIO configuration up to covering $30 \%$ of the total interface area results in a $7.5 \%$ temperature reduction for the bottom die.

Not only the amount of dummy $\mu$ bumps is important, but also the location: the point $(\rho, T)=\left(0.2,103^{\circ} C\right)$ is achieved considering a single horizontal rectangular $\mu$ bump array in the center of the interface layer. Since the array is not aligned with the hot areas, its effect is much smaller than the one obtained with the same $\rho$ value but placing the $\mu$ bump arrays above the heat dissipation region. For the same amount of $\mu$ bumps, the temperature reduction can be 30 times higher.

Comparison with FEM results shows a maximum error for these data of $1.3 \%$. This is really low but it has to be taken into account if different configurations want to be compared: conclusions, concerning if a specific design is better than another, can be drawn only if the difference is higher than $2-3 \%$.

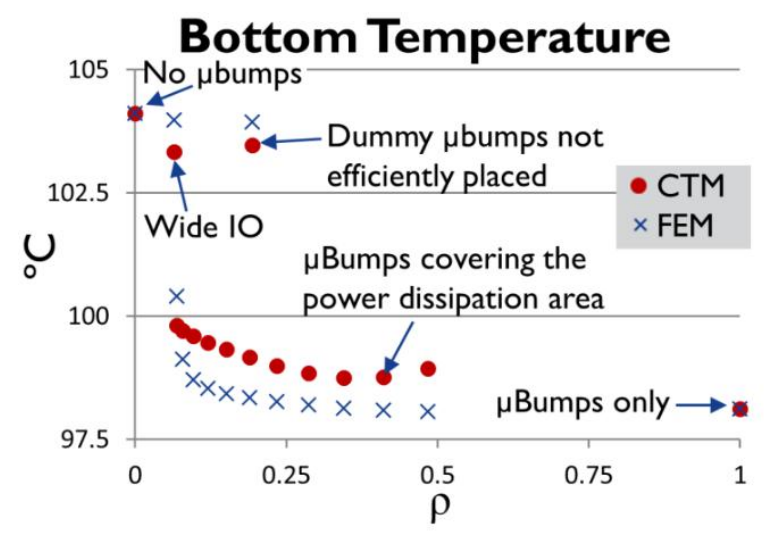

Fig. 11 Bottom die temperature, in the location indicated in Fig. 10 , as a function of the $\mu$ bump area ratio $\rho$. Red circles refer to CTM results while blue crosses to FEM ones. Significant cases are highlighted. 
It should be noted that this analysis, to assess the best amount and placement of dummy $\mu$ bumps in order to fulfil certain technological requirements while keeping the fabrication costs low, can be quickly and easily performed by just changing entries in the binary $\mu$ bump matrix. Once this input operation has been performed, less than 10 seconds are needed to obtain the temperature profiles for the specific configuration.

\section{CONCLUSIONS}

The capability of a novel CTM approach to easily, quickly and accurately analyze the steady state thermal impact of the interface layer in two dies stacks has been discussed in the paper. The effect on the temperature profiles of different material properties and of different $\mu$ bump configurations has been demonstrated to be detectable by the CTM by just changing input numbers and entries in matrices. The results are obtained quickly (30 times faster than FEM), with high accuracy $(<2 \%$ error with respect to FEM) and without requiring particular modeling expertise. The model is, therefore, suitable to be used in the early design phase of 3D-ICs when numerous configurations need to be compared and tested with the aim of meeting specific technical requirements while keeping production costs low.

The presented CTM is based on the Green's function theory applied through convolution and FFT. Due to the finite stack size and the material heterogeneity in the interface layer, correction steps have to be introduced. The method of images is used to deal with the insulating lateral boundary conditions while a novel correction methodology is employed to take into account the specific interface structure. This methodology is based on fitting models of various FEM results of simplified situations in which uniform power is dissipated on one die and insulation is imposed on one side; generalizations for more generic structures and power dissipations followed. The CTM has been validated against FEM results of identical structures showing error smaller than $2 \%$ and a 30 times speed-up.

\section{REFERENCES}

[1] B. Wang and P. Mazumder, "Accelerated Chip-Level Thermal Analysis Using Multilayer Green's Function," Computer-Aided Design of Integrated Circuits and Systems, IEEE Transactions on, vol. 26, no. 2 , pp. 325-344, 2007.

[2] De Vos, J., et al. "High density $20 \mu \mathrm{m}$ pitch CuSn microbump process for high-end 3D applications," Electronic Components and Technology Conference (ECTC), 2011 IEEE 61st. IEEE, 2011.

[3] Milojevic, D, et al. "DRAM-on-logic Stack - Calibrated thermal and mechanical models integrated into PathFinding flow", IEEE Custom Integrated Circuits Conference (CICC), 2011.

[4] Milojevic, D. et al.; "PathFinding and TechTuning", Three Dimensional System Integration, Springer (2011), pp. $137-186$.

[5] W. Huang, et al. "HotSpot: A compact thermal modeling methodology for early-stage VLSI design," Very Large Scale Integration (VLSI) Systems, IEEE Transactions on vol. 14, no.5, pp. 501-513, 2006.

[6] T.Y. Wang, and C.C.P Chen. "3-D thermal-ADI: A lineartime chip level transient thermal simulator," Computer-
Aided Design of Integrated Circuits and Systems, IEEE Transactions on, vol. 21, no. 12, pp. 1434-1445, 2002.

[7] A. Sridhar, et al. "3D-ICE: a Compact Thermal Model for Early-Stage Design of Liquid-Cooled ICs," Computers, IEEE Transactions on, accepted for publication.

[8] E.G.T. Bosch and M.N. Sabry. "Thermal compact models for electronic systems," Semiconductor Thermal Measurement and Management, 2002. Eighteenth Annual IEEE Symposium, pp. 21-29, 2002.

[9] L. Choobineh, and J. Ankur. "Analytical Solution for Steady-State and Transient Temperature Fields in Vertically Stacked 3-D Integrated Circuits," Components, Packaging and Manufacturing Technology, IEEE Transactions on, vol. 28, no. 4, pp.2031-2039, 2012.

[10] L. Codecasa. "A novel approach for generating boundary condition independent compact dynamic thermal networks of packages," Components and Packaging Technologies, IEEE Transactions on, vol. 28, no. 4, pp. 593604, 2005.

[11] B. Barabadi, et al. "Rapid multi-scale transient thermal modeling of packaged microprocessors using hybrid approach," Electronics Packaging Technology Conference (EPTC), 2012 IEEE 14th, pp. 157-164, 2012.

[12] D. Celo, et al. "The creation of compact thermal models of electronic components using model reduction," $\mathrm{Ad}$ vanced Packaging, IEEE Transactions on, vol. 28, no. 2, pp. 240-251, 2005.

[13] A. Augustin, and T. Hauck. "A New Approach to Boundary Condition Independent Compact Dynamic Thermal Models," Semiconductor Thermal Measurement and Management Symposium, 2007. SEMI-THERM 2007. Twenty Third Annual IEEE, pp. 228-232, 2007.

[14] V.M. Hériz, et al. "Method of images for the fast calculation of temperature distributions in packaged VLSI chips," Thermal Investigation of ICs and Systems, 2007. THERMINIC 2007. 13th International Workshop on, pp. 18-25, 2007.

[15] J. Park, "Fast Thermal Analysis of Vertically Integrated Circuits (3D ICs) Using Power Blurring Method," InterPACK, vol. 9, pp. 19-23, 2009.

[16] A. Ziabari, and A. Shakouri. "Fast thermal simulations of vertically integrated circuits (3D ICs) including thermal vias," Thermal and Thermomechanical Phenomena in Electronic Systems (ITherm), 2012 13th IEEE Intersociety Conference on, pp. 588-596, 2012.

[17] B. Wang, and P. Mazumder. "Accelerated chip-level thermal analysis using multilayer Green's function," Computer-Aided Design of Integrated Circuits and Systems, IEEE Transactions on, vol. 26, no. 2, pp. 325-344, 2007.

[18] Y. Zhan, and S. Sapatnekar. "High-efficiency Green function-based thermal simulation algorithms," ComputerAided Design of Integrated Circuits and Systems, IEEE Transactions on, vol. 26, no. 9, pp.1661-1675, 2007.

[19] F. Maggioni, et al. "Convolution based Compact Thermal Model for 3D-ICs: Methodology and Accuracy Analysis," Thermal Investigation of ICs and Systems, 2013. THERMINIC 2013. 19th International Workshop on, pp. 152-157, 2013.

[20] I. Özdemir, et al. "Computational homogenization for heat conduction in heterogeneous solids," International 
journal for numerical methods in engineering, vol. 73, no. 2, pp. 185-204, 2008.

[21] F. Maggioni, et al. "Convolution Based Steady State Compact Thermal Model for 3D-ICs: Methodology for Including the Thermal Impact of Die to Die Interconnections", 15th International Heat Transfer Conference
(IHTC-15). Abstract accepted, pending final paper acceptance.

[22] JESD229, "Wide I/O Single Data Rate (Wide I/O SDR)", Nov. 2011, http://www.jedec.org/standards-documents/results/jesd229. 